\title{
Dynamics of the Davydov-Scott monomer in a thermal bath: Comparison of the full quantum and semiclassical approaches
}

\author{
J. Cuevas, ${ }^{1}$ P. A. S. Silva, ${ }^{2}$ F. R. Romero, ${ }^{1}$ and L. Cruzeiro ${ }^{2}$ \\ ${ }^{1}$ Grupo de Física No Lineal, Universidad de Sevilla, Departamento de Física Aplicada I, Escuela Universitaria Politécnica, \\ C/ Virgen de África, 7, 41011 Sevilla, Spain \\ ${ }^{2}$ CCMAR and FCT, University of Algarve, Campus de Gambelas, 8000 Faro, Portugal
}

(Received 21 March 2007; published 12 July 2007)

\begin{abstract}
The quantum state diffusion equation is applied to the problem of energy transfer in proteins. Lindblad operators, capable of coupling the full quantum Davydov-Scott monomer to a thermal bath, are derived. Numerical simulations with the QSD equation show that the Lindblad operators derived do recreate the exact equilibrium ensemble for the Davydov-Scott monomer. Comparison of the results obtained with the full quantum and with the semiclassical systems shows that, at biological temperatures, the latter provides a good approximation of the former.
\end{abstract}

DOI: 10.1103/PhysRevE.76.011907

PACS number(s): 87.14.Ee, 03.65.Yz

\section{INTRODUCTION}

The possibility that protein function involves the creation and transfer of vibrational excited states was first put forward in a classical paper by McClare [1]. This suggestion was then taken up by Davydov who was interested in muscle contraction [2]. In a series of studies, Davydov proposed that these vibrational excited states are amide I vibrations and that they can be stabilized by the interaction with local vibrations of the peptide group, thus affecting, and being affected by, the hydrogen bond length between peptide groups in a protein [3]. The analytical studies of Davydov on continuum models of $\alpha$ helices were extended to numerical simulations of discrete models by Scott and co-workers (for a review of the whole field until 1992 see Ref. [4]). The general conclusion from these studies was that, at low temperatures, the amide I excitation is localized and induces a shortening of the neighboring hydrogen bonds, a state that bears strong resemblances with the polaron $[7,8]$ and that has been designated in the literature as the Davydov soliton. Proteins, however, must function at biological temperatures, and to be useful, the Davydov soliton must survive at these temperatures. The Davydov-Scott model includes two types of vibration: an intrasite vibration, the amide I vibration and intersite vibrations, the vibrations of the amide sites as a whole. Many of the studies assume that the latter vibrations can be treated classically, an approximation that we shall refer to here as semiclassical. The first simulations at finite temperature were performed within the semiclassical approximation and effected a coupling of the classical part of the system to a classical bath [9]. The result was that the Davydov soliton dispersed in a few picoseconds at biological temperatures. This result clashed with others, obtained later by Monte Carlo simulations of the full quantum system (QMC), which showed that, at thermal equilibrium, the local distortion induced by the presence of an amide I excitation increases with temperature [10]. This conflict was resolved by showing that, in a semiclassical system, the coupling of the classical part to a classical bath leads to a classical behavior for the quantum part as well [11]. Thus, while the states of a classical amide I excitation, as described by the Davydov-Scott model, are predominantly delocalized at finite temperature, the states of a quantum amide I excitation in the same conditions are predominantly localized [12]. A set of dynamic equations, within the semiclassical approximation, was proposed [11] that leads to the same equilibrium averages as the QMC simulations at biological temperatures. While at low temperatures, these equations lead to localized amide I excitations that travel in an almost coherent fashion along protein $\alpha$ helices, as in the low temperature studies $[3,4]$, at biological temperatures, although still localized, the amide I excitations jump stochastically from site to site, changing their shape and their velocity as they travel $[11,13]$. These finite temperature dynamical equations couple the stationary Schrödinger equation for the amide I excitations to a Langevin equation for the lattice and thus are only truly valid when the amide I excitations are much faster than the lattice motions [13]. In a recent study, the finite temperature dynamical equations in Refs. [11,13] were applied to amide I energy transfer in a real protein [14]. As it is not possible to treat all the degrees of freedom of a protein quantum mechanically, it is important to assess the accuracy of the dynamical equations in Refs. [11,13]. The main purpose of this study is to assess the degree of accuracy of the semiclassical model, and of the specific set of equations proposed in Refs. [11,13], with respect to the full quantum model, particularly in what concerns dynamics at finite temperature. To simulate the quantum dynamics at finite temperature we will use the Quantum State Diffusion (QSD) theory [15]. QSD has been developed to describe open quantum systems and finds applications in phenomena such as the crossing of the quantum to the classical domain, decoherence and measurement processes. Here we will apply it to the study of the influence of a thermal bath on a quantum system. Since the number of variables needed to represent quantum states increase with the power of the number of amide sites, the comparison we make here is for the simplest possible Davydov-Scott Hamiltonian, representing just one peptide group (one site for amide I excitations), hydrogen bonded to a nonexcitable group. The paper is organized as follows. In Sec. II the theoretical framework to deal with finite temperature dynamics in full quantum systems is developed. In Sec. III results ob- 
tained in the semiclassical and in the full quantum approaches are presented and the paper ends with a discussion in Sec. IV.

\section{FINITE TEMPERATURE DYNAMICS OF THE DAVYDOV MONOMER}

The Hamiltonian for the full quantum Davydov monomer is $\hat{H}_{\mathrm{q}}=\hbar \omega\left(\hat{a}^{\dagger} \hat{a}+\frac{1}{2}\right)+\chi^{\prime} u \hat{a}^{\dagger} \hat{a}+\frac{1}{2} \kappa \hat{u}^{2}+\frac{\hat{p}^{2}}{2 M}$, where $\hat{a}^{\dagger}(\hat{a})$ is the creation (annihilation) operator for an amide excitation, $\hat{u}$ is the operator for the displacement from the equilibrium position of the amide site, $\hat{p}$ is the operator for the momentum of the amide site as a whole, $\hbar \omega$ is the energy of an amide I excitation, $\chi^{\prime}$ is the variation of the amide I energy with changes in the hydrogen bond length, $\kappa$ is the elasticity of amide I site, and $M$ is the mass of the amide site. Here we adopt the values that have been most used in the literature, namely, $\hbar \omega=1660 \mathrm{~cm}^{-1}, \chi^{\prime}=62 \mathrm{pN}, \kappa=13 \mathrm{~N} / \mathrm{m}$, and $M$ $=114$ a.m.u. [4]. In terms of the operator $\hat{b}=\sqrt{\frac{M \Omega}{2 \hbar}} \hat{u}$ $+1 \sqrt{\frac{1}{2 M \hbar \Omega}} \hat{p}$, the previous Hamiltonian can be written as

$$
\hat{H}_{\mathrm{q}}=\hbar \omega\left(\hat{a}^{\dagger} \hat{a}+\frac{1}{2}\right)+\chi\left(\hat{b}^{\dagger}+\hat{b}\right) \hat{a}^{\dagger} \hat{a}+\hbar \Omega \hat{b}^{\dagger} \hat{b},
$$

where $\chi=\chi^{\prime} \sqrt{\hbar /(2 M \Omega)}$ and $\Omega=\sqrt{\kappa / M}$.

The Hamiltonian (1) only includes an extrinsic anharmonicity, due to the interaction of the amide I excitation with the hydrogen bond that connect the carbonyl groups, $\mathrm{C}=\mathrm{O}$ to the amine groups $\mathrm{H}-\mathrm{N}$. Although multiquanta amide I states have a certain degree of intrinsic anharmonicity, as has been established experimentally [5] and considered theoretically [6], we do not include it here because we restrict ourselves to one amide I quantum states only. This is because the sources for amide I vibrations we envisage here are biological processes such as the hydrolysis of ATP, which release, at most, energy for two amide I excitations. In future work we will consider also two-quanta energy states.

We are mainly interested in what happens at finite temperature. Let us first consider the thermal equilibrium situation. Because at biological temperatures the amide I energy is approximately $8 k_{B} T$, thermalizing the whole system, that is, both the amide I vibrations and the lattice displacements, at these temperatures or below, leads to zero amide I quanta, on average, and an amide site that is decoupled from the lattice, which fluctuates as a harmonic oscillator. We are not interested in this trivial total thermalization. What we want to know is the dynamics of the lattice when one amide I quantum is present. The Hamiltonian (1) conserves the number of amide I excitations and thus the thermal average of the number of amide I excitations is specified by the initial condition that we consider. On the other hand, the Hamiltonian (1) does not conserve the number of quanta in the lattice, which fluctuates, at finite temperature. To determine the thermal equilibrium averages for the full quantum system, under the conditions just described, we note that the Hamiltonian (1) can be diagonalized by the Lee, Low, and Pines transformation [16], $\hat{R}=\exp \left[\frac{\chi}{\hbar \Omega}\left(\hat{b}^{\dagger}-\hat{b}\right) \hat{a}^{\dagger} \hat{a}\right]$, a procedure that leads to the transformed Hamiltonian $\widetilde{H}$ :

$$
\tilde{H}=\hbar \omega\left(\hat{A}^{\dagger} \hat{A}+\frac{1}{2}\right)+\hbar \Omega\left(\hat{B}^{\dagger} \hat{B}+\frac{1}{2}\right)-\frac{\chi^{2}}{\hbar \Omega}\left(\hat{A}^{\dagger} \hat{A}\right)^{2},
$$

where the transformed operators $\hat{A}$ and $\hat{B}$ are given by

$$
\hat{A}=\hat{R}^{\dagger} \hat{a} \hat{R}=\hat{a} \exp \left[\frac{\chi}{\hbar \Omega}\left(\hat{b}^{\dagger}-\hat{b}\right)\right], \quad \hat{B}=\hat{R}^{\dagger} \hat{b} \hat{R}=\hat{b}+\frac{\chi}{\hbar \Omega} \hat{a}^{\dagger} \hat{a} .
$$

It is readily verified that $\hat{a}^{\dagger} \hat{a}=\hat{A}^{\dagger} \hat{A}$, so that these two operators have the same eigenvectors and eigenvalues. Using the diagonalized Hamiltonian (2), it is easy to show that the thermal averages over lattice fluctuations, for the full quantum system, are

$$
\left\langle\left\langle\hat{n}_{b}\right\rangle\right\rangle=\nu_{B E}+\left(\frac{\chi n}{\hbar \Omega}\right)^{2}, \quad\langle\langle\hat{u}\rangle\rangle=-\frac{\chi^{\prime} n}{\kappa},
$$

where $\hat{n}_{b}=\hat{b}^{\dagger} \hat{b}, n$ is the number of amide I excitations, $\nu_{B E}$ $=1 /\left[\exp \left(\hbar \Omega / k_{B} T\right)-1\right]$ is the Bose-Einstein distribution, and $\langle\langle\cdots\rangle\rangle$ stands for equilibrium averages over thermal fluctuations of the lattice.

The corresponding thermal equilibrium values for a semiclassical Hamiltonian, in which the lattice displacement and momentum are real, are

$$
\left\langle\left\langle n_{b}\right\rangle\right\rangle=\frac{k_{B} T}{\hbar \Omega}+\left(\frac{\chi n}{\hbar \Omega}\right)^{2}, \quad\langle\langle u\rangle\rangle=-\frac{\chi^{\prime} n}{\kappa},
$$

where $k_{B}$ is Boltzmann's constant. Comparing Eq. (4) with Eq. (5) shows that the equilibrium average number of quanta in the semiclassical lattice only differs from the full quantum value because of the first term. However, as expected, the semiclassical thermal average number of lattice quanta (5) tends to the quantum expression (4) as temperature increases. At biological temperatures, i.e., for $T=37{ }^{\circ} \mathrm{C}, k_{B} T / \hbar \Omega$ $\approx 4.9$, while the full quantum Bose-Einstein distribution $\nu_{\mathrm{BE}} \approx 4.4$, corresponding to a relative error of $11 \%$. Moreover, the average displacements from the equilibrium position are exactly the same in the full quantum regime and in the semiclassical regime, at all temperatures.

Let us now consider the non-equilibrium situation. For the full quantum system we resort to the QSD equation [15]

$$
\begin{aligned}
|d \psi\rangle= & -\frac{1}{\hbar} \hat{H}|\psi\rangle d t+\sum_{j}\left(\hat{L}_{j}-\left\langle\hat{L}_{j}\right\rangle\right)|\psi\rangle d \xi_{j} \\
& +\frac{1}{2} \sum_{j}\left(2\left\langle L_{j}^{\dagger}\right\rangle \hat{L}_{j}-\hat{L}_{j}^{\dagger} \hat{L}_{j}-\left\langle\hat{L}_{j}^{\dagger}\right\rangle\left\langle\hat{L}_{j}\right\rangle\right)|\psi\rangle d t,
\end{aligned}
$$

where $\hat{H}$ is given by Eq. (1), $|\psi\rangle$ is the wave function of the full quantum system, $\hat{L}_{j}$ are the Lindblad operators [17] that represent the influence of the environment on the system, and $\left\langle\hat{L}_{j}\right\rangle=\left\langle\psi\left|\hat{L}_{j}\right| \psi\right\rangle$. In Eq. (6) the first term describes the deterministic evolution of the system, while the two other terms describe its interaction with the thermal bath. $d \xi_{j}$ are independent normalized complex stochastic variables with a zero mean and a zero covariance [15]. The Lindblad operators $\hat{L}_{j}$ are not unique and can be defined by the Lindblad 
form of the master equation for the system [17]

$$
\frac{d \hat{\rho}}{d t}=-\frac{1}{\hbar}[\hat{H}, \hat{\rho}]+\sum_{j}\left(\hat{L}_{j} \hat{\rho} \hat{L}_{j}^{\dagger}-\frac{1}{2} \hat{L}_{j}^{\dagger} \hat{L}_{j} \hat{\rho}-\frac{1}{2} \hat{\rho} \hat{L}_{j}^{\dagger} \hat{L}_{j}\right),
$$

where $\hat{\rho}$ is the density operator of the system. For a harmonic oscillator, the thermalization process can be achieved by the action of two Lindblad operators with the following form: $\hat{L}_{1}=\sqrt{\gamma\left(1+\nu_{\mathrm{BE}}\right)} \hat{b}, \hat{L}_{2}=\sqrt{\gamma \nu_{\mathrm{BE}}} \hat{b}^{\dagger}$ [18], where $\gamma$ is an arbitrary parameter related to the intensity of the heat bath, i.e., the larger the $\gamma$, the stronger the interaction with the bath and the faster the relaxation to thermal equilibrium. As explained above, the thermalization we are interested in is that in which the number of amide I quanta is conserved, while the number of lattice quanta is allowed to fluctuate. To derive Lindblad operators capable of thermalizing the Davydov-Scott Hamiltonian (1) in this way we resort to a heuristic reasoning. The diagonalized Hamiltonian $\widetilde{H}(2)$ is linear in the lattice operators $\hat{B}$, so, in terms of $\hat{B}$, the Lindblad operators are the same as for the harmonic oscillator. Using the relationship between $\hat{B}$ and $\hat{b}$ given by Eq. (3), we get the following Lindblad operators for our system:

$$
\begin{gathered}
\hat{L}_{1}=\sqrt{\gamma\left(1+\nu_{\mathrm{BE}}\right)} \hat{B}=\sqrt{\gamma\left(1+\nu_{\mathrm{BE}}\right)}\left(\hat{b}+\frac{\chi}{\hbar \Omega} \hat{a}^{\dagger} \hat{a}\right), \\
\hat{L}_{2}=\sqrt{\gamma \nu_{\mathrm{BE}}} \hat{B}^{\dagger}=\sqrt{\gamma \nu_{\mathrm{BE}}}\left(\hat{b}^{\dagger}+\frac{\chi}{\hbar \Omega} \hat{a}^{\dagger} \hat{a}\right) .
\end{gathered}
$$

With the latter Lindblad operators the bath is constituted by phonons with the same discrete energy levels as the lattice.

Having obtained the Lindblad operators that thermalize the full quantum Davydov-Scott Hamiltonian, we can derive the quantum dynamical equations at finite temperature by making use of the fact that the time dependent expression for the thermal average of a given operator $\hat{A}$, and of its derivative, are obtained from the time dependent density matrix using the relations $\langle\hat{A}(t)\rangle=\operatorname{Tr}[\hat{\rho}(\mathrm{t}) \hat{A}]$ and $\langle d \hat{A}(t) / d t\rangle$ $=\operatorname{Tr}(\mathrm{d} \hat{\rho} / \mathrm{dt} \hat{A})$. Using the latter expressions together with Eqs. (7)-(9), leads to the following differential equations for the thermal averages of $\hat{n}_{b}(t)$ and $\hat{b}(t)$ :

$$
\begin{aligned}
& \frac{d\left\langle\hat{n}_{b}\right\rangle}{d t}=-i \frac{\chi n}{\hbar}\left(\left\langle\hat{b}^{\dagger}\right\rangle-\langle\hat{b}\rangle\right)-\gamma\left\langle\hat{n}_{b}\right\rangle-\gamma \frac{\chi n}{2 \hbar \Omega}\left(\left\langle\hat{b}^{\dagger}\right\rangle\right. \\
&+\langle\hat{b}\rangle)+\gamma \nu_{\mathrm{BE}} \\
& \frac{d\langle\hat{b}\rangle}{d t}=i \frac{\chi n}{\hbar}-\left(i \Omega+\frac{\gamma}{2}\right)\langle\hat{b}\rangle-\gamma \frac{\chi n}{2 \hbar \Omega} .
\end{aligned}
$$

In the latter equations and in what follows $\langle\cdots\rangle$ denotes thermal average of the quantity in the square parenthesis and $\langle\langle\cdots\rangle\rangle$, which was used in Eqs. (4) and (5), denotes its thermal equilibrium value.

\section{RESULTS}

\section{A. Averages}

One problem in comparing the full quantum approach with the semiclassical approach is that the states of the quantum lattice are represented by a wave function and there can be many wave functions that, when averaged over the displacement and momentum operators, lead to the same values of the displacement and momentum. Thus, one well-defined initial value problem in the semiclassical system can be mapped into a multitude of equivalent initial value problems in the corresponding quantum system. To minimize this problem we choose initial values of zero for the lattice displacement and momentum. With this choice, the solutions to the full quantum dynamical Eqs. (10), (11) are

$$
\begin{gathered}
\left\langle\hat{n}_{b}(t)\right\rangle=\left\langle\left\langle\hat{n}_{b}\right\rangle\right\rangle+\left[2\left(\frac{\chi n}{\hbar \Omega}\right)^{2}-\left\langle\left\langle\hat{n}_{b}\right\rangle\right\rangle\right] e^{-\gamma t / 2} \\
-2\left(\frac{\chi^{n}}{\hbar \Omega}\right)^{2} \cos (\Omega t) e^{-\gamma t / 2}, \\
\langle\hat{u}(t)\rangle=-\frac{\chi^{\prime} n}{M \Omega^{2}}\left[1-\cos (\Omega t) e^{-\gamma t / 2}\right], \\
\langle\hat{p}(t)\rangle=-\frac{\chi^{\prime} n}{\Omega} \sin (\Omega t) e^{-\gamma t / 2} .
\end{gathered}
$$

It is readily verified that equations (12)-(14) lead to the correct thermal equilibrium averages (4) for the respective variables. Thus, the Lindblad operators (8) and (9) do allow for a correct thermalization of the quantum Davydov-Scott Hamiltonian (1) in that they lead to the exact quantum thermal equilibrium values.

In the semiclassical approximation $[11,13]$, the nonequilibrium equations for the Davydov-Scott monomer are reduced to a single stochastic equation for the classical lattice displacement $u$, in which the number $n$ of amide I excitations, appears as a factor in a constant driving force

$$
M \ddot{u}+M \Omega^{2} u+M \gamma \dot{u}=F(t)-\chi^{\prime} n,
$$

where $F(t)$ is a stochastic variable that represents the classical bath and its time correlation obeys the fluctuationdissipation theorem $\left\langle F\left(t_{1}\right) F\left(t_{2}\right)\right\rangle=2 M \gamma K_{B} T \delta\left(t_{1}-t_{2}\right)$. The $\delta$ function in time means that the stochastic forces $F(t)$ are Markovian. In this way, the nature of the bath is the same in the semiclassical and in the quantum systems. For $\chi^{\prime}=0$, Eq. (15) describes a Brownian harmonic oscillator [19]. For $\chi^{\prime}$ $\neq 0$ we have a shifted Brownian harmonic oscillator, for which the oscillations take place not around zero, but around the thermal equilibrium average (4), as in the full quantum system. Two different dynamical regimes can be distinguished: overdamped (for $\gamma>2 \Omega$ ) and underdamped (for $\gamma$ $<2 \Omega$ ). In both the semiclassical and the quantum approaches, we concentrate on the underdamped regime (for $\gamma<2 \Omega$ ), more interesting because the system undergoes intrinsic oscillations. Also, a small $\gamma$ corresponds to a weak coupling to the thermal bath, something that minimizes the effect of the Markovian nature of the stochastic forces. 
Another problem that arises when comparing the dynamics, at finite temperature, predicted by the two approximations, are the different representations of the thermal bath in each approximation. Indeed, inspection of Eqs. (15) and (6) shows that while thermalization of the semiclassical system involves one real stochastic variable, the thermalization of the corresponding quantum system involves four independent real stochastic variables. For this reason, we first compare the time evolution of thermal averages in the two approximations and only consider individual trajectories later. Initial states for the classical lattice are specified by two real values: its displacement and its momentum. With the same initial conditions as before, integration of Eq. (15) leads to the following solutions:

$$
\begin{aligned}
&\left\langle n_{\mathrm{b}}(t)\right\rangle= \frac{k_{B} T}{\hbar \Omega}\left[1-\frac{\gamma^{2}}{4 \beta^{2}} \cos (\beta t) \mathrm{e}^{-\gamma t / 2}-\frac{\Omega^{2}}{\beta^{2}} e^{-\gamma t}\right] \\
&+\left(\frac{\chi n}{\hbar \Omega}\right)^{2}\left[1-2\left(\cos (\beta t)+\frac{\gamma}{2 \beta} \sin (\beta t)\right) e^{-\gamma t / 2}\right. \\
&\left.+\left(1+\frac{\gamma^{2}}{2 \beta^{2}} \sin ^{2}(\beta t)+\frac{\gamma}{2 \beta} \sin (2 \beta t)\right) e^{-\gamma t}\right], \\
&\langle u(t)\rangle=-\frac{\chi^{\prime} n}{M \Omega^{2}}\left[1-\left(\cos (\beta t)+\frac{\gamma}{2 \beta} \sin (\beta t)\right) e^{-\gamma t / 2}\right] \\
&\langle p(t)\rangle=-\frac{\chi^{\prime} n}{\Omega^{2}}\left(\beta+\frac{\gamma^{2}}{4 \beta}\right) \sin (\beta t) e^{-\gamma t / 2}
\end{aligned}
$$

where $\beta \equiv \sqrt{\Omega^{2}-\gamma^{2} / 4}$. We see that, for $t \rightarrow \infty$, all variables tend to their correct thermal equilibrium values, given by Eq. (5). Also, in the limit $\Omega \gg \gamma / 2$, the semiclassical displacement and momentum tend to the full quantum expressions (12)-(14). Thus, when comparing the two regimes we concentrate on the variable that differs most in the two regimes, namely, $\left\langle\hat{n}_{b}(t)\right\rangle$.

Figure 1 shows the dependence of the cumulative average of $\left\langle n_{\mathrm{b}}(t)\right\rangle$, which is defined as $C\left(\left\langle n_{b} c(t)\right\rangle\right)=1 / t \int_{0}^{t}\left\langle n_{b}(\tau)\right\rangle d \tau$, for $T=10$ and $310 \mathrm{~K}$. This figure displays this dependence for the full quantum and semiclassical system together with the result of the integration of the QSD equation, using the program written by Schack and Brun [20]. Both at 10 and $310 \mathrm{~K}$, we see that the averages obtained with the QSD equation (dotted line) are approximately on top of the exact analytical quantum results (solid line). On the other hand, we conclude that, at $T=10 \mathrm{~K}$, quantum effects of the lattice are important and the semiclassical system is a poor approximation to the full quantum system. For the more biologically relevant temperature $T=310 \mathrm{~K}$, the quantum effects of the lattice are much less important and the semiclassical system leads approximately to the same time evolution as the full quantum system.

As explained above, a direct comparison of single trajectories at finite temperature in the two regimes is precluded by the different ways in which the coupling to the thermal bath is made in each regime. However, it is of interest to know what the single trajectories look like, especially for the full
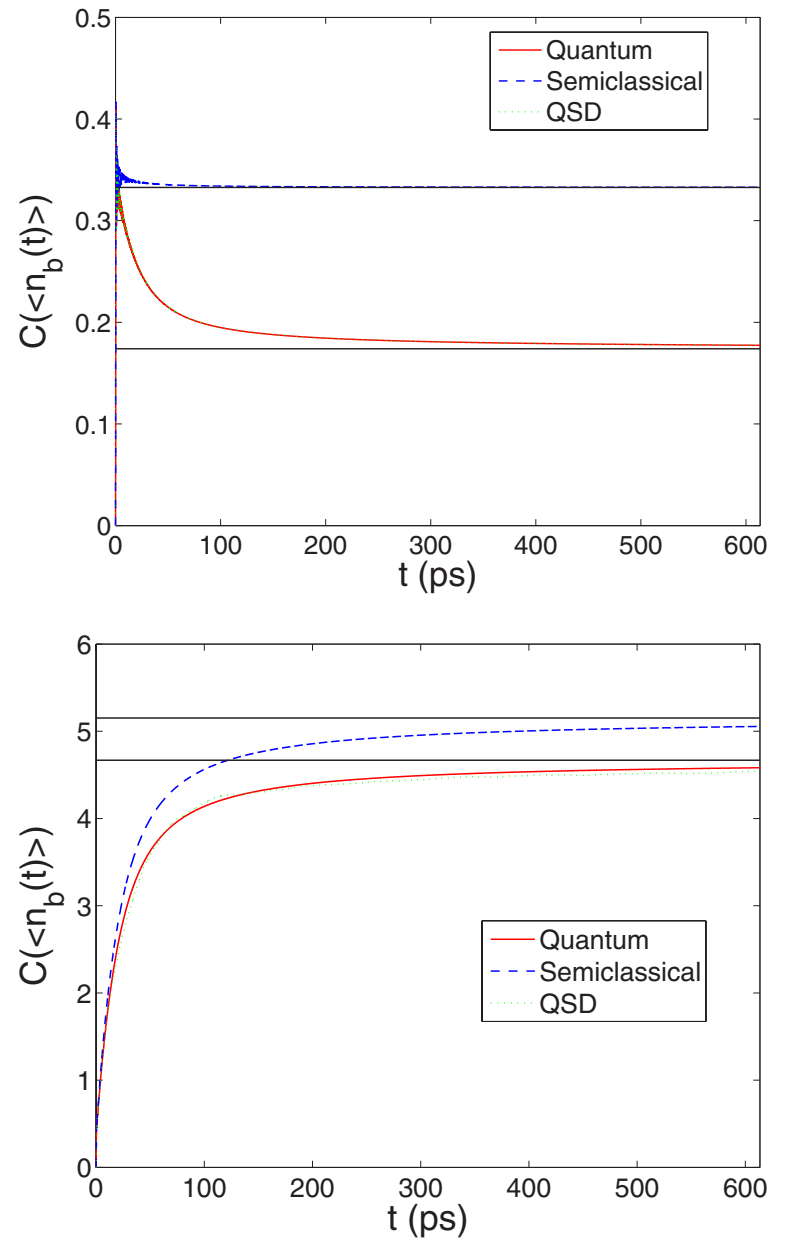

FIG. 1. (Color online) Time evolution of $C\left(\left\langle\hat{n}_{b}(t)\right\rangle\right)$ for the full quantum system (solid line) and for the semiclassical system (dashed line), for $\gamma=0.01 \Omega$ and $n=1$. The horizontal lines represent the corresponding thermal equilibrium averages. The dotted line was obtained numerically using the QSD program written by Schack and Brun [20] and represents an average over 500 individual trajectories, with a basis set with 200 levels for the lattice quanta and a time step of $0.01 / \Omega$. Top panel corresponds to $T=10 \mathrm{~K}$, and bottom panel to $T=310 \mathrm{~K}$. The QSD line is not visible as it is almost coincident with the Quantum line.

quantum system. Such single trajectories are displayed in Fig. 2 for the time evolution of the lattice displacement and for the number of quanta obtained in the full quantum system, from the numerical integration of the QSD equation (6) (solid line), and in the semiclassical system, from the integration of Eq. (15) (dashed line), at $T=10 \mathrm{~K}$ and $T=310 \mathrm{~K}$. Also shown in Fig. 2 is a trajectory obtained with the QSD equation (6) for $\chi=0$, in which case the Davydov-Scott monomer reduces to a simple harmonic oscillator with frequency $\Omega$, uncoupled to the amide I vibrations. As expected, while the Davydov-Scott monomer oscillates around a displaced equilibrium position, the harmonic oscillator oscillates around zero. The oscillations of all three systems have the frequency $\Omega$ of the lattice and, in spite of the differences between the coupling to the thermal baths in the two regimes, the oscillations in the semiclassical system are approximately in phase with the oscillations in the quantum 

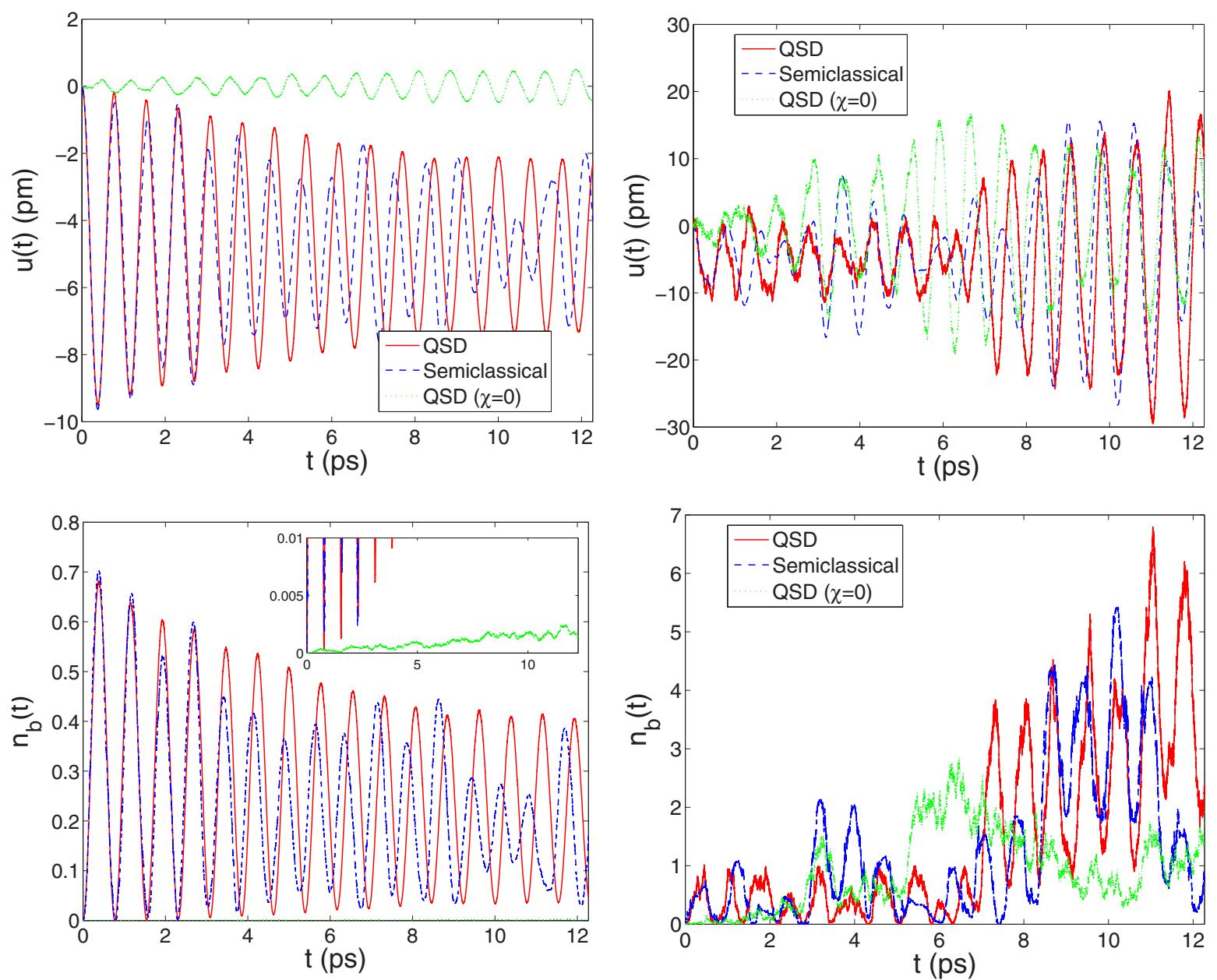

FIG. 2. (Color online) Time evolution of the lattice displacement (upper row) and the number of lattice quanta (bottom row), for a single trajectory, in the full quantum system (solid line) and in the semiclassical system (dashed line), as well as for the harmonic oscillator (dotted line), when $T=10 \mathrm{~K}$ (left) and when $T=310 \mathrm{~K}$ (right). The inset at the bottom left is a zoom up around $\hat{n}_{b}=0$.

system for the first four cycles. At $310 \mathrm{~K}$ temperature, dephasing occurs faster, as expected, but recurrences are also visible. Comparing with the oscillations of the simple harmonic oscillator it becomes apparent, particularly at low temperatures, that the Davydov-Scott monomer extracts energy from the bath at a much higher rate. Indeed, the number of lattice quanta at $T=310 \mathrm{~K}$ for $\chi=0$ (dotted line) is generally below that for $\chi \neq 0$ (solid line) and for $T=10 \mathrm{~K}$, the number of lattice quanta for $\chi=0$ is so small that it is practically on top of the horizontal axis, as can be seen in the inset at the bottom left of Fig. 2. All figures show that, even at biological temperatures, when the noise becomes significant, the quantum beats of the lattice, induced by the mere presence of an amide I excitation and enhanced by the thermal bath, are clearly visible.

\section{B. Standard deviations}

A variable of great interest when comparing quantum and classical approximations is the standard deviation. Let us first consider the standard deviations $\sigma_{u}$ and $\sigma_{p}$ of $\hat{u}$ and $\hat{p}$, respectively, where $\sigma_{A}=\left\langle A^{2}\right\rangle-\langle A\rangle^{2}$ is calculated in the full quantum regime. To this end we need the value of $\left\langle\hat{u}^{2}\right\rangle$ and $\left\langle\hat{p}^{2}\right\rangle$, which are given by

$$
\begin{gathered}
\left\langle\hat{u}^{2}\right\rangle=\frac{\hbar}{2 m \Omega}\left(\left\langle\hat{b}^{\dagger} \hat{b}^{\dagger}\right\rangle+\langle\hat{b} \hat{b}\rangle+2\left\langle\hat{n}_{b}\right\rangle+1\right) \\
\left\langle\hat{p}^{2}\right\rangle=-\frac{m \hbar \Omega}{2}\left(\left\langle\hat{b}^{\dagger} \hat{b}^{\dagger}\right\rangle+\langle\hat{b} \hat{b}\rangle-2\left\langle\hat{n}_{b}\right\rangle-1\right),
\end{gathered}
$$

where $\langle\hat{b} \hat{b}\rangle$ obeys the following differential equation:

$$
\frac{d\langle\hat{b} \hat{b}\rangle}{d t}=-(2 i \Omega+\gamma)\left(\langle\hat{b} \hat{b}\rangle-\frac{\chi n}{\hbar \Omega}\langle\hat{b}\rangle\right)
$$

$\langle\hat{b}\rangle$ being the solution to the differential equation (11) with initial condition $\langle b(0)\rangle=0$, given by

$$
\langle\hat{b}(t)\rangle=\frac{\chi n}{\hbar \Omega}\left(\mathrm{e}^{-i \Omega t-\gamma t / 2}-1\right) .
$$

Introducing this solution in Eq. (21) we get 


$$
\langle\hat{b} \hat{b}\rangle=\left(\frac{\chi n}{\hbar \Omega}\right)^{2}\left(1-e^{-i \Omega t-\gamma t / 2}\right)+C e^{-2 i \Omega t-\gamma t}
$$

in which $C$ is a complex constant.

In order to determine the value of $C$ we need to know the initial values of $\sigma_{u}$ and $\sigma_{p}$ (which are the same as for the harmonic oscillator), together with their equilibrium values. They are as follows [21]:

$$
\begin{gathered}
\sigma_{u}(0)=\frac{\hbar}{2 M \Omega}, \quad \sigma_{p}(0)=\frac{\hbar M \Omega}{2}, \\
\sigma_{u}(\infty)=\frac{\hbar}{2 M \Omega} \operatorname{coth} \frac{\hbar \Omega}{2 K_{B} T}, \quad \sigma_{p}(\infty)=\frac{\hbar M \Omega}{2} \operatorname{coth} \frac{\hbar \Omega}{2 K_{B} T} .
\end{gathered}
$$

With these values it can be shown that the complex constant $C=\chi n /(\hbar \Omega)$ and that the quantum standard deviations of $\hat{u}$ and $\hat{p}$ become

$$
\begin{aligned}
& \sigma_{u}(t)=\frac{\hbar}{2 M \Omega}\left[2 \nu_{\mathrm{BE}}\left(1-e^{-\gamma t}\right)+1\right], \\
& \sigma_{p}(t)=\frac{M \hbar \Omega}{2}\left[2 \nu_{\mathrm{BE}}\left(1-e^{-\gamma t}\right)+1\right] .
\end{aligned}
$$

These results, that do not depend on $\chi$, are the same as for the harmonic oscillator [21].

On the other hand, it can be shown that in the semiclassical approximation, the standard deviations $\sigma_{u}^{c}$ and $\sigma_{p}^{c}$ have the following expressions:

$$
\begin{aligned}
\sigma_{u}^{c}(t)= & \frac{\gamma K_{B} T}{2 M \beta^{2}}\left[-\left(\frac{\gamma}{2 \Omega^{2}} \cos (\beta t)+\frac{\beta}{\Omega^{2}} \sin (\beta t)\right) \exp (-\gamma t / 2)\right. \\
& \left.-\frac{2}{\gamma} \exp (-\gamma t)+\frac{2 \beta^{2}}{\gamma \Omega^{2}}\right], \\
\sigma_{p}^{c}(t)= & \frac{M \gamma K_{B} T}{2 \beta^{2}}\left[-\left(\frac{\gamma}{2} \cos (\beta t)-\beta \sin (\beta t)\right) \exp (-\gamma t / 2)\right. \\
& \left.-\frac{2 \Omega^{2}}{\gamma} \exp (-\gamma t)+\frac{2 \beta^{2}}{\gamma}\right] .
\end{aligned}
$$

As was found in the full quantum regime, the standard deviations in the semiclassical regime do not depend on $\chi$. In the limit $\Omega \gg \gamma / 2$, their expressions can be approximated by

$$
\begin{gathered}
\sigma_{u}(t)=\frac{\hbar \nu_{\mathrm{MB}}}{M \Omega}\left(1-e^{-\gamma t}\right), \\
\sigma_{p}(t)=M \hbar \Omega \nu_{\mathrm{MB}}\left(1-e^{-\gamma t}\right) .
\end{gathered}
$$

The latter equations only differ from those obtained in the full quantum regime Eqs. (26) and (27) for two reasons: first, the quantum expressions include a term associated with the uncertainty principle that states that the standard deviations cannot both be zero, even for well defined initial conditions, and, secondly, because where in the quantum expressions we have the Bose-Einstein distribution $\nu_{\mathrm{BE}}$ in the semiclassical
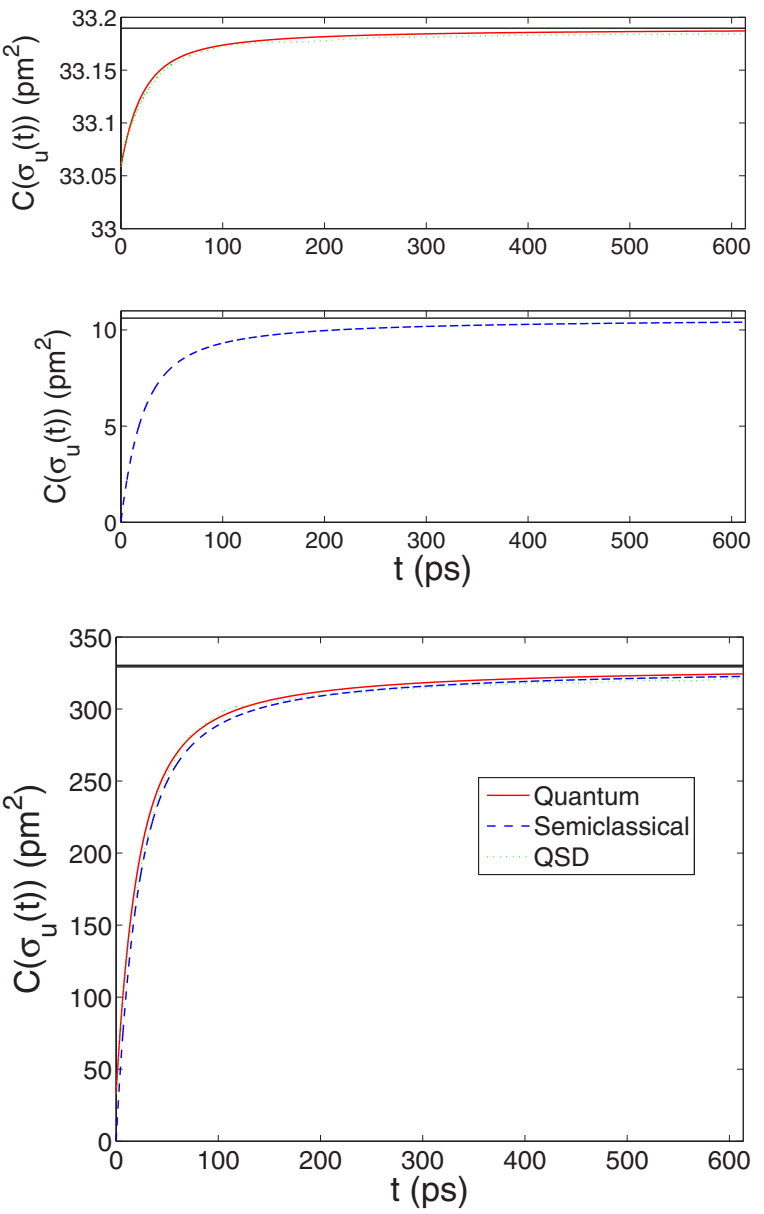

FIG. 3. (Color online) Time evolution of $C\left[\sigma_{u}(t)\right]$ for the full quantum system (solid line) and for the semiclassical system (dashed line), for $\gamma=0.01 \Omega$ and $n=1$. The horizontal lines represent the corresponding thermal equilibrium averages. The dotted line was obtained numerically using the QSD program [20]. Top panel corresponds to $T=10 \mathrm{~K}$, and bottom panel to $T=310 \mathrm{~K}$.

expressions we have the classical number $\nu_{\mathrm{MB}}=\frac{k_{B} T}{\hbar \Omega}$.

The time evolution of the cumulative standard deviation is shown in Fig. 3. As was observed in Fig. 1, the semiclassical approximation is worse at $T=10 \mathrm{~K}$, where the relative error in the standard deviation of the displacement is approximately $67 \%$, than at $T=310 \mathrm{~K}$, where that error is virtually zero, something that lends further support for the lack of importance of quantum effects of the lattice at biological temperatures.

\section{DISCUSSION}

As far as we know, this is the first time that the QSD equation is applied to the problem of energy transfer in proteins and that Lindblad operators, capable of providing a correct thermalization of the Davydov-Scott system, are derived. Our main purpose is to make use of this full quantum formalism to evaluate the validity, at finite temperature, of the semiclassical approximation of the Davydov-Scott model. The relatively small system considered here allowed us to derive analytical expressions to important physical 
variables, in an exact manner. A general conclusion is that, at $T=10 \mathrm{~K}$, quantum effects on the lattice cannot be neglected. On the other hand, at biological temperatures, the semiclassical Davydov-Scott monomer does provide a good approximation to the corresponding full quantum system.

While the comparison that was made here is not possible to make for the large system that even a small real protein is, the fact that only a single site for amide I excitations was considered constitutes nevertheless a limitation of the present study. More extended systems may behave differently. Indeed, in a previous study of an extended Davydov-Scott system with 50 amide I sites [12], in which only thermal equilibrium averages were considered, it was found that, at $T$ $=0.7 \mathrm{~K}$, there was a $15 \%$ relative error in the semiclassical thermal equilibrium lattice displacement correlated with the position of the excitation, which disappeared above $10 \mathrm{~K}$. On the other hand, for the one amide I site studied here the thermal equilibrium value of the lattice displacement is the same in the semiclassical and in the full quantum systems [see Eqs. (4) and (5)], at all temperatures (i.e., the relative error of the semiclassical thermal equilibrium lattice displacement is zero). In a forthcoming publication we will consider the system with two amide I sites, where the possibility of jumps of the amide I excitation from one site to another, which is likely to be responsible for the slightly different results obtained in more extended systems, is also included.

It should also be said that the ultimate aim of this work is to understand how proteins work. Following McClare [1], Davydov [2,3], and Scott [4], the main assumption is that protein function involves the storing of energy in the form of vibrational excited states, something that was recently designated as the VES hypothesis [14]. In recent years, experimental evidence for the feasibility of this hypothesis has been accumulating. Indeed, while an early objection to a role of vibrational excited states in bioenergetics was their short lifetimes, thought to be in the subpicosecond range [1], it is now known that, even in isolated amino acids, the lifetime of the amide I mode can last approximately 1 ps and the first measurements of the lifetime of this mode in real proteins showed that, in myoglobin, the higher energy side of the amide I band has a lifetime of $15 \mathrm{ps}$ [22]. More recently, a low-lying band of amide I has also been found in myoglobin [23]. This state, which is thought to arise from self-trapping, has a longer lifetime, of $30 \mathrm{ps}$ at $50 \mathrm{~K}$, than the normal amide I band, which is 5 ps [23]. On the other hand, Hamm and co-workers monitored NH excitations in a model $\alpha$-helix and not only find a self-trapped state for the NH stretch vibration in this system, but also that it can only arise when the helical structure is intact [24], i.e., when the $\mathrm{C}=\mathrm{O}$ groups are hydrogen bonded to each other, as is assumed in the Davydov-Scott model. Furthermore, experiments in the group of Hamm [5,25-27] also confirm the results obtained in previous computer simulations $[11,13]$ according to which at low temperature vibrational excitations are self-trapped, while at biological temperatures they are localized because of static and dynamical disorder. Finally, experiments in the group of Hochstrasser [28] show that amide I vibrations can jump from one helix to another, as happens in computer simulations [14], and as it must happen in real proteins if the VES hypothesis is valid. Thus, in spite of some perceived difficulties [29] it can be said that the recent experimental evidence supports the kind of interactions first postulated by Davydov [3] for amide I excitations in proteins and encourages the continuation of the work in this direction [29]. It is thus important to make more accurate theoretical calculations that can lead to the quantitative predictions necessary to establish whether, in fact, proteins, in cells, do make use of vibrational excited states while performing their functions. The work in this paper constitutes a step in this direction.

\section{ACKNOWLEDGMENTS}

J.C. and F.R.R. acknowledge financial support from the MECD Project No. FIS2004-01183. The Fundação para a Ciência e a Tecnologia, Portugal, is also acknowledged for the financial support to P.A.S.S.
[1] C. W. F. McClare, Ann. N.Y. Acad. Sci. 227, 74 (1974).

[2] A. S. Davydov, J. Theor. Biol. 38, 559 (1973).

[3] A. S. Davydov, J. Theor. Biol. 66, 379 (1977); Int. J. Quantum Chem. 16, 5 (1979); Biology and Quantum Mechanics (Pergamon, New York, 1982).

[4] A. Scott, Phys. Rep. 217, 1 (1992).

[5] J. Edler and P. Hamm, J. Chem. Phys. 117, 2415 (2002).

[6] V. Pouthier, Phys. Rev. E 68, 021909 (2003).

[7] T. Holstein, Ann. Phys. (N.Y.) 8, 325 (1959).

[8] T. Holstein, Ann. Phys. (N.Y.) 8, 343 (1959).

[9] P. S. Lomdahl and W. C. Kerr, Phys. Rev. Lett. 55, 1235 (1985).

[10] X. Wang, D. W. Brown, and K. Lindenberg, Phys. Rev. Lett. 62, 1796 (1989).

[11] L. Cruzeiro-Hansson, Europhys. Lett. 33, 655 (1996).

[12] L. Cruzeiro-Hansson and V. M. Kenkre, Phys. Lett. A 203, 362 (1995).
[13] L. Cruzeiro-Hansson and S. Takeno, Phys. Rev. E 56, 894 (1997).

[14] L. Cruzeiro, J. Phys.: Condens. Matter 17, 7833 (2005).

[15] I. Percival, Quantum State Diffusion (Cambridge University Press, Cambridge, 1998).

[16] T. D. Lee, F. E. Low, and D. Pines, Phys. Rev. 90, 297 (1953).

[17] G. Lindblad, Commun. Math. Phys. 48, 119 (1976).

[18] V. V. Dodonov, S. S. Mizrahi, and A. L. de S. Silva, J. Opt. B: Quantum Semiclassical Opt. 2, 271 (2000).

[19] S. Chandrasekhar, Rev. Mod. Phys. 15, 1 (1943); G. E. Uhlenbeck and L. S. Ornstein, Phys. Rev. 36, 823 (1930).

[20] R. Schack and T. A. Brun, Comput. Phys. Commun. 102, 210 (1997).

[21] A. Isar, A. Sandulescu, and W. Scheid, Physica A 322, 233 (2003).

[22] A. Xie, L. van Der Meer, W. Hoff, and R. H. Austin, Phys. Rev. Lett. 84, 5435 (2000). 
[23] R. H. Austin, A. Xie, L. V. Meer, M. Shinn, and G. Neil, J. Phys.: Condens. Matter 15, S1693 (2003).

[24] J. Edler, R. Pfister, V. Pouthier, C. Falvo, and P. Hamm, Phys. Rev. Lett. 93, 106405 (2004).

[25] J. Edler, P. Hamm, and A. C. Scott, Phys. Rev. Lett. 88, 067403 (2002).

[26] J. Edler and P. Hamm, J. Chem. Phys. 119, 2709 (2003).
[27] J. Edler and P. Hamm, Phys. Rev. B 69, 214301 (2004).

[28] C. Fang, A. Senes, L. Cristian, W. F. DeGrado, and R. M. Hochstrasser, Proc. Natl. Acad. Sci. U.S.A. 103, 16740 (2006).

[29] P. A. Lindgard and A. M. Stoneham, J. Phys.: Condens. Matter 15, V5 (2003) 